\title{
Hacia una medicina de excelencia en México: el protocolo Código Infarto, una visión desde la bioética traslacional
}

\author{
Gabriela Borrayo-Sánchez, ${ }^{1}$ Abelardo Flores-Morales, ${ }^{2}$ Leonardo Salas-Collado $y$ \\ Myriam M. Altamirano-Bustamante ${ }^{3 *}$ \\ ${ }^{1}$ Programa A Todo Corazón, Código Infarto; ${ }^{2}$ Hospital de Cardiología; ${ }^{3}$ Unidad de Investigación en Enfermedades Metabólicas, Grupo Transfuncional \\ en Ética Clínica. Instituto Mexicano del Seguro Social, Centro Médico Nacional Siglo XXI, Ciudad de México, México
}

\section{Resumen}

Introducción: México es el país con mayor mortalidad por infarto agudo de miocardio con elevación del segmento ST (IAM CEST), por lo que el Instituto Mexicano del Seguro Social desarrolló el protocolo de atención para los servicios de urgencias denominado Código Infarto. En este artículo se discuten aspectos de la medicina traslacional con una perspectiva bioética e integral. Objetivo: Analizar el protocolo Código Infarto desde la perspectiva de la bioética traslacional. Método: Se realizó una aproximación centrada en el problema a través del equilibrio reflexivo, así como la aplicación del método integral para el discernimiento ético. Resultados: El protocolo de atención para los servicios de urgencias Código Infarto se rige por la medicina basada en la evidencia y la medicina basada en valores; se orienta por el principio de integridad que considera las seis dimensiones de la calidad para la atención de pacientes con IAM CEST. Conclusión: El protocolo supera algunos determinantes sociales adversos que afectan la atención médica del IAM CEST, disminuye la mortalidad, la carga económica global de la enfermedad y desarrolla una medicina de excelencia de alto alcance social.

PALABRAS CLAVE: Código Infarto. Infarto agudo de miocardio. Bioética traslacional. Dilemas éticos.

Towards medicine of excellence in Mexico: the "Código Infarto" protocol, a view from the perspective of translational bioethics

\begin{abstract}
Introduction: Mexico is the country with the highest mortality due to ST-elevation acute myocardial infarction (STEMI), and the IMSS has therefore developed the protocol of care for emergency departments called Código Infarto (Infarction Code). In this article, aspects of translational medicine are discussed with a bioethical and comprehensive perspective. Objective: To analyze the Código Infarto protocol from the perspective of translational bioethics. Method: A problem-centered approach was carried out through reflective equilibrium (or Rawls' method), as well as by applying the integral method for ethical discernment. Results: The protocol of care for emergency services Código Infarto is governed by evidence-based medicine and value-based medicine; it is guided by a principle of integrity that considers six dimensions of quality for the care of patients with STEMI. Conclusion: The protocol overcomes some adverse social determinants that affect STEMI medical care, reduces mortality and global economic disease burden, and develops medicine of excellence with high social reach.
\end{abstract}

KEY WORDS: Código Infarto. Acute myocardial infarction. Translational bioethics. Ethical dilemmas.

Correspondencia:

${ }^{*}$ Myriam M. Altamirano-Bustamante

E-mail: biocatalisismma@gmail.com

0016-3813/C 2020 Academia Nacional de Medicina de México, A.C. Publicado por Permanyer. Este es un artículo open access bajo la licencia CC BY-NC-ND (http://creativecommons.org/licenses/by-nc-nd/4.0/).
Fecha de recepción: 26-02-2020

Fecha de aceptación: 12-05-2020

DOI: 10.24875/GMM.20000090
Gac Med Mex. 2020;156:372-378

Disponible en PubMed

www.gacetamedicademexico.com 


\section{Introducción}

En 2017, México fue considerado el país con la más alta mortalidad por infarto agudo de miocardio con elevación del segmento ST (IAM CEST): $27.5 \%$ en comparación con $7.5 \%$ del promedio registrado por la Organización para la Cooperación y el Desarrollo Económicos (OCDE) ese año, respecto al cual el Instituto Nacional de Estadística y Geografía reportó 141619 defunciones por enfermedades cardiovasculares. Lo anterior es relevante si se considera que los recursos económicos son limitados: en 2017, en México solo $5.5 \%$ del producto interno bruto se destinó a salud y se reportaron 2.4 médicos por mil habitantes, a diferencia de 3.4 por mil habitantes señalados por la OCDE; además, el gasto per cápita fue cuatro veces menor al promedio. ${ }^{1-3}$ Existen otros determinantes sociales que afectan la atención sanitaria como la falta de accesibilidad, la heterogeneidad de las normas sociales e institucionales y la inequidad en la prestación de los servicios; ${ }^{4}$ son escasas las políticas de salud que mejoran la atención y trasladan sus resultados a una reducción de la mortalidad.

Algunos modelos de gestión integral han reducido la mortalidad mediante protocolos de atención, los cuales son un puente entre la medicina basada en evidencia y la medicina basada en valores (mejores prácticas aplicadas en la atención). ${ }^{5}$ Facilitar la transición de la investigación a una aplicación clínica con beneficios en la salud se le conoce como medicina traslacional y permite el libre tránsito de conocimientos para afrontar los retos actuales en salud. ${ }^{6}$ El objetivo de este estudio es analizar, desde la perspectiva de la bioética traslacional, el protocolo de atención para los servicios de urgencias denominado Código Infarto, ya que su implementación ha logrado traducir los conocimientos científicos en reducción de la mortalidad en pacientes con infarto agudo de miocardio con elevación de segmento ST (IAM CEST). ${ }^{7}$

\section{Código Infarto}

El protocolo Código Infarto inició su implementación en el Instituto Mexicano del Seguro Social desde 2015, se enfoca al diagnóstico y reperfusión del IAM CEST e incluye dos de siete ejes del programa de atención integral A Todo Corazón. ${ }^{7}$ El objetivo es garantizar el diagnóstico y tratamiento del paciente que demanda atención de urgencias por IAM CEST, de manera que reciba reperfusión miocárdica durante los primeros minutos de una forma más eficiente (accesible, sistematizada, precisa y humanista). ${ }^{7}$ El protocolo considera los principios metodológicos clínicos de las estrategias de salud, ${ }^{8}$ con un carácter institucional e integral; ${ }^{9}$ se centra en el monitoreo de las actividades críticas para lograr sus objetivos, así como en la medición de indicadores del proceso de gestión, entre los cuales están la reducción del tiempo de atención desde los primeros síntomas y del tiempo para la aplicación de algún mecanismo de reperfusión, con terapia fibrinolítica o intervención coronaria percutánea. En ambos casos se han reducido significativamente (de 92 a 72 minutos [ $p=0.004$ ] y de 144 a 90 minutos [p < 0.0001], respectivamente). ${ }^{10}$ Lo anterior ha sido posible gracias a la capacitación educativa presencial y en línea del personal de primer contacto, la comunicación efectiva entre los tres niveles de atención y el uso de sistemas de la información en salud, como el Centro Regulador de Código Infarto, que integra y organiza la información de nuevos casos. Otra herramienta disponible para la población es la app IMSS digital. ${ }^{11}$ Además se suma la rehabilitación cardiaca temprana desde las primeras 24 a 48 horas. Con ello, Código Infarto ha logrado reducir los días de incapacidad: de 67.7 a 58.6 días, $p<0.0001 .{ }^{12}$

Finalmente, el Registro Nacional de Síndrome Coronario Agudo del Instituto Mexicano del Seguro Social es el estudio de "mundo real" más grande del país y de la mayoría de los países latinoamericanos; se registraron 15981 individuos con diagnóstico de IAM CEST, quienes fueron incluidos en el protocolo Código Infarto. ${ }^{13}$ Se observó una reducción significativa de los casos no reperfundidos (65.2 a $29.6 \%$, $p<0.0001$ ), principalmente por incremento de la intervención coronaria percutánea (9.4 a $31.3 \%$ ) y la terapia fibrinolítica (25.5 a $40.1 \%, p<0.0001$ ), en la cual, además, se demostró reducción de $35 \%$ en el tiempo de aplicación (92 minutos contra 60 minutos).$^{13}$ Por primera vez en México, la mejora en la reperfusión repercutió en una reducción significativa de la mortalidad: de 28 a $9.4 \%{ }^{1}$ (Figura 1).

\section{Método}

Se realizó un análisis del protocolo de atención para los servicios de urgencias Código Infarto mediante el método integral, para el discernimiento bioético, y el método de equilibrio reflexivo, para el contraste y esclarecimiento de juicios relacionados con los aspectos epistémicos, éticos, legales y médicos. ${ }^{14}$ Como se muestra en la Figura 2, ambos intervienen en el proceso traslacional: experimental (T1), clínico (T2), operativo (T3) y estratégico o de políticas de salud (T4); y en seis fases 


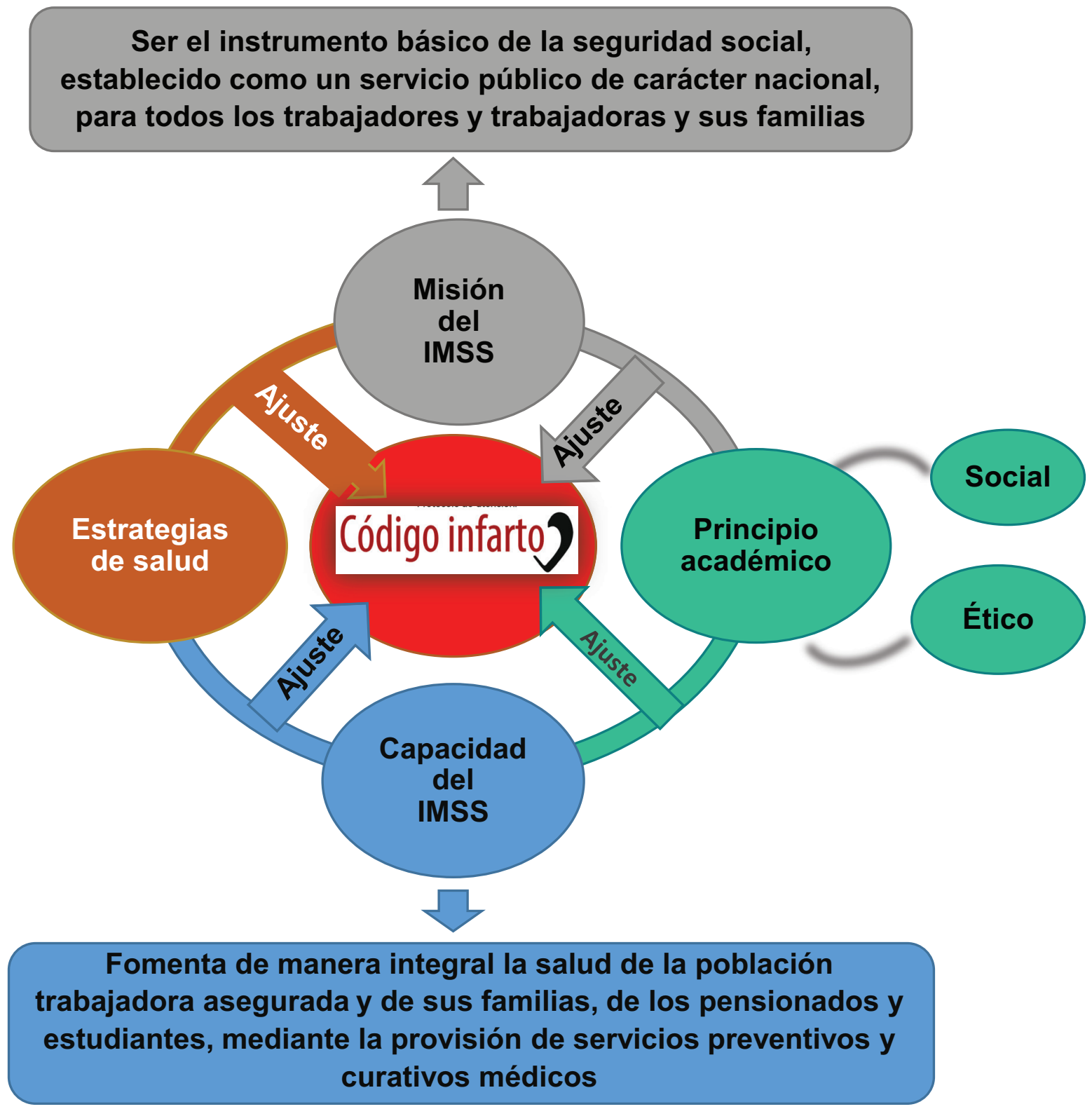

Figura 1. Discernimiento ético del protocolo Código Infarto del Instituto Mexicano del Seguro Social.

de aplicación del conocimiento: generación (F1), implementación (F2), confirmación clínica (F3), validación de prioridades (F4), desarrollo de nuevas guías de práctica clínica (F5) y elaboración de políticas de salud (F6). ${ }^{15}$

\section{Resultados}

\section{Discernimiento bioético del protocolo Código Infarto}

En la actualidad, la medicina de precisión o personalizada representa un gran reto debido a su complejidad y demanda; sin embargo, con el fortalecimiento del binomio medicina basada en evidencias-medicina basada en valores ${ }^{16}$ se parte de las múltiples dimensiones de la persona, del personal sanitario y de la red de apoyo social (elementos esenciales del tratamiento integral en el proceso de salud-enfermedad), lo que se facilita con la medicina traslacional, con la finalidad última de mejorar la calidad de la salud de las personas. Lo anterior lleva a la práctica de una medicina de excelencia. ${ }^{17}$

En México, la seguridad social es heterogénea, fraccionada en diferentes instituciones de salud, con 


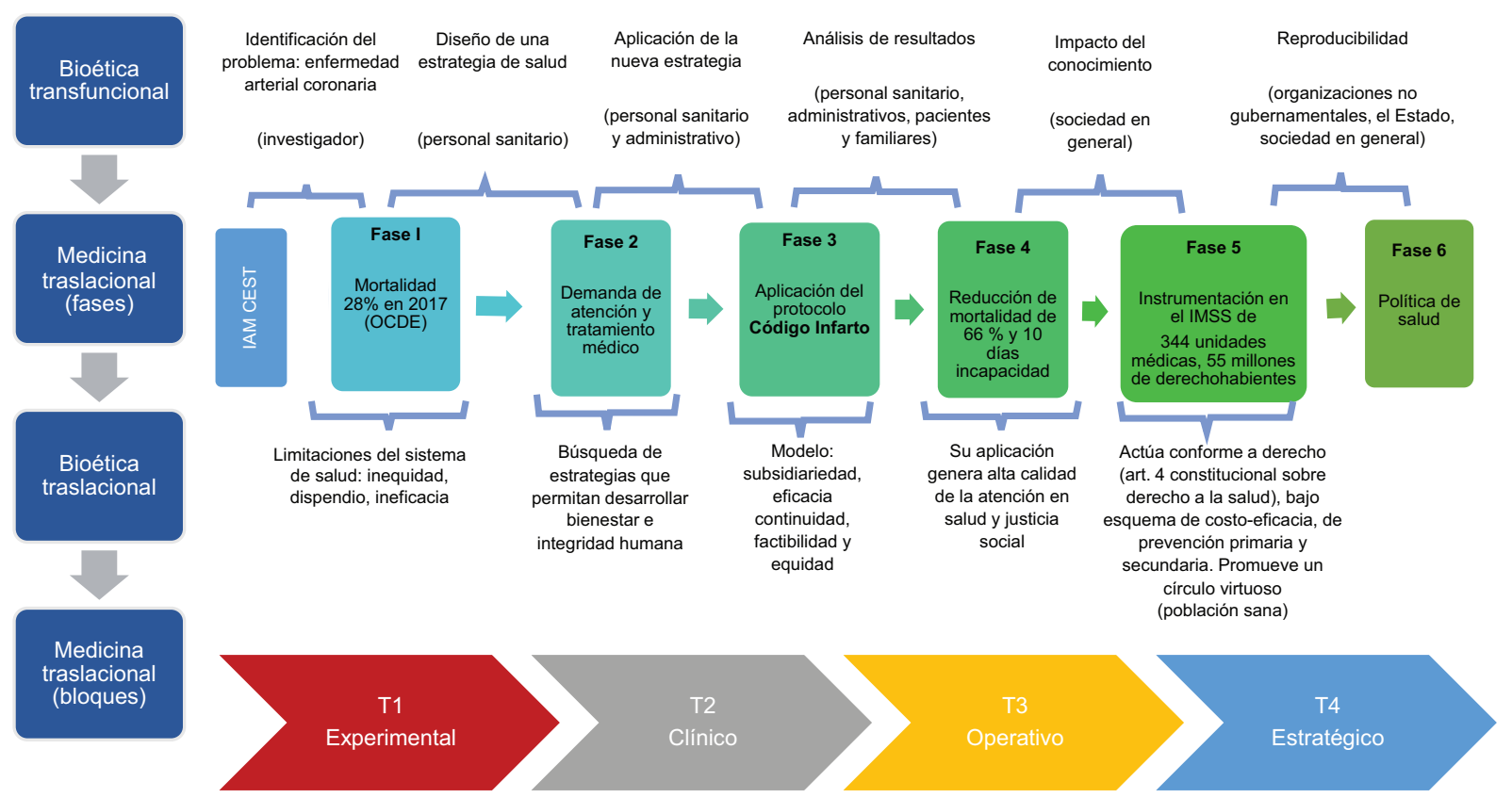

Figura 2. Proceso traslacional del protocolo Código Infarto desde una perspectiva ética. IMSS = Instituto Mexicano del Seguro Social.

la aspiración del "mayor bien posible" a los asegurados antes, durante y al término de las acciones de salud, sobre todo las que llegan a millones de personas. ${ }^{18}$ Código Infarto se sustenta en la visión organizacional funcionalista de Burrel, que parte de una realidad medible y comparte aspectos como continuidad, factibilidad y equidad, en busca del beneficio de quien padece la enfermedad, privilegiando una cardiología integral. ${ }^{19}$

\section{Aplicación del método integral}

El método integral es el ideal, un método de análisis de máximos con el que se busca el "bien" del paciente. Integra los tres aspectos de la acción: el agente moral, la acción misma (basada en derechos y obligaciones) y las consecuencias de la acción. Analiza el "bien" desde varias perspectivas éticas. Cual una bailarina de ballet que tiene un pie en la Tierra, representa las diferentes corrientes éticas determinadas por las circunstancias empíricas, pero con el pie extendido en el aire con elegancia, como signo de flexibilidad, busca nuevos principios éticos que perfeccionen al profesional de la salud en su relación con el paciente.

A través de cinco preguntas, este método permite la deliberación, la reflexión crítica y la funcionalidad, al incluir a todo el equipo de salud, al paciente y a los familiares, permitiendo al médico tratante tomar una decisión responsable, es decir, con conocimiento, voluntad y libertad, desplegando su máximo poder moral. ${ }^{20,21}$

1. ¿Cuál es el hecho?

En México, la reperfusión miocárdica ocurría en menos de $50 \%$ de las ocasiones, con el protocolo Código Infarto aumentó su frecuencia y con ello se redujo la mortalidad por IAM.

2. ¿Cuál "bien" se busca?

Un sistema reproducible que promueva la seguridad y bienestar integral del paciente, con una ética de máximos que permita desarrollar una "ética floreciente" del personal de salud en beneficio del paciente.

3. ¿Quiénes son los beneficiarios del "bien"? Los pacientes, familiares, médicos, la institución y la sociedad en general.

4. ¿Quiénes son los actores principales?

El paciente afectado por la enfermedad que pone en riesgo su vida, el personal de salud que tiene la responsabilidad ética, los familiares y millones de derechohabientes que pertenecen al Instituto Mexicano del Seguro Social.

5. ¿Cómo se identifican y estratifican los dilemas éticos?

Dado que el derecho a la reperfusión es limitado en México, las estrategias de salud requieren la suma de valores y su puesta en práctica, por lo que nos planteamos el siguiente cuestionamiento: 
¿de qué forma Código Infarto permite transitar hacia una medicina de excelencia?

a) En cuanto al aspecto aretológico: el programa convoca al personal de salud y a la sociedad a un estado de consciencia solidaria para beneficiar al paciente con IAM CEST, promueve virtudes, estrecha las diferencias económicas y sociales y salvaguarda el desarrollo integral de pacientes y médicos.

b) En cuanto al aspecto deontológico: las instituciones de salud velan por el cumplimiento del artículo 4 constitucional, el cual indica que "toda persona tiene derecho a un medio ambiente sano para su desarrollo y bienestar". Código Infarto nace en el seno del Instituto Mexicano del Seguro Social, cuya misión es la protección de la seguridad social.

c) En cuanto al aspecto utilitarista: el tratamiento del IAM CEST incrementa los años de vida ganados y su calidad; además, disminuye el tiempo en el diagnóstico y tratamiento, con la reducción de días de estancia e incapacidad laboral.

Código Infarto busca el "bien mayor" y a la vez preservar la integridad de la persona, con acciones correctas y consecuencias efectivas y rentables.

\section{El proceso traslacional de Código Infarto}

La medicina traslacional utiliza los descubrimientos científicos básicos y clínicos para generar herramientas orientadas al diagnóstico de la enfermedad y su tratamiento. ${ }^{22}$ Por otra parte, la bioética transfuncional y la traslacional permiten la comunicación basada en la confianza y el respeto entre expertos. ${ }^{23}$ Ambas pretenden que las normas y valores guíen hacia prácticas biomédicas éticamente aceptables del fenómeno vital, con una reflexión crítica, radical y universal por parte de un grupo multidisciplinario que genera ideas, conocimientos, conceptos, metodologías, experiencia e instrumentos para resolver un problema común de manera integral, con la transferencia efectiva del análisis de la estructura axiológica de las prácticas biomédicas al ámbito médico (cama del paciente, consulta externa y quirófanos, entre otros) y social ${ }^{24}$ (Figura 2).

\section{Código Infarto como política de salud y de justicia en la práctica clínica}

La salud es un bien común y prioritario para los países. La OCDE propuso a México construir un sistema de salud equitativo, eficiente y sostenible; ofrecer un cuidado de la salud continuo, personalizado, proactivo, con el desarrollo de estándares y directrices. ${ }^{25}$ En este sentido, el artículo 4 constitucional, el artículo 2 de la Ley General de Salud y la Ley del Instituto Mexicano del Seguro Social disponen que la protección de la salud tiene como finalidad el bienestar físico y mental de la persona para contribuir al ejercicio pleno de sus

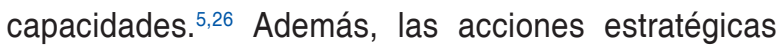
enfocadas a políticas de salud deben ser el vínculo social entre el bienestar y la colectividad.

Con lo anterior se busca la justicia, entendida en el sentido aristotélico como la virtud perfecta, bajo sus dos formas: la justicia distributiva, cuya finalidad consiste en regular la distribución de bienes y activos en la comunidad; y la justicia conmutativa, que regula la relación entre particulares. La justicia social añade dinamismo a la justicia legal y resalta la justicia distributiva; además, asegura el respeto y la promoción de los derechos para todos. ${ }^{27}$ Es así como se encamina hacia una justicia como capacidad, la cual considere a las personas y promueva su florecimiento y máximo potencial (Figura 3).

\section{Discusión}

La desigualdad es un obstáculo para que un país pueda alcanzar el máximo de sus potencialidades. En México, $44.4 \%$ de la población vive en la pobreza (Consejo Nacional de Evaluación de la Política de Desarrollo Social, 2018) ${ }^{28} y$, en contraste, $75 \%$ tiene obesidad o sobrepeso, por lo que la política social oscila entre el defecto y el exceso. Ahí estriba la importancia de reconocer la trascendencia de la responsabilidad individual y colectiva en materia de salud.

En el mundo existen esfuerzos para elevar la calidad de la atención del IAM CEST, tales como el programa Infarctus du Myocarde, en Francia, que utiliza indicadores de eficacia y seguridad; ${ }^{29}$ el protocolo Code STEMI, en Canadá, en operación las 24 horas del día: ${ }^{30}$ y Lifeline, para la atención rápida del IAM CEST, de la Asociación Americana del Corazón. ${ }^{31}$ Con Código Infarto, México es uno de los pioneros en el protocolo de atención de urgencias del IAM CEST. Su normativa dota de fuerza y dirección a los participantes, genera consciencia de las regulaciones, deja claro que su omisión solamente retrasa la atención médica y amplifica las complicaciones en los pacientes que pueden ser beneficiados con la aplicación del protocolo.

Sin embargo, estas estrategias no se han analizado desde la bioética traslacional, vector de cambio, la 


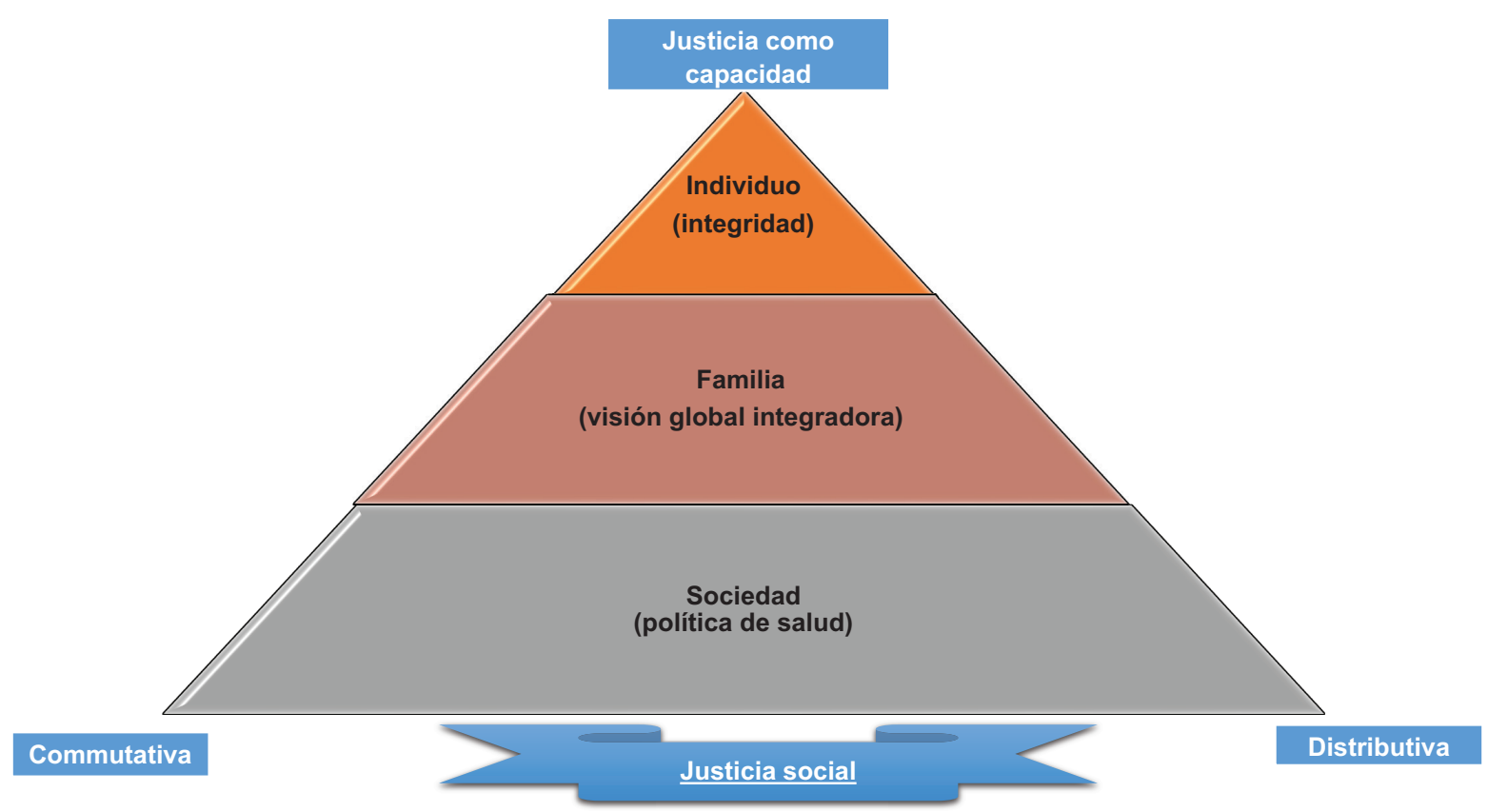

Figura 3. Elementos y dinámica de la pirámide de la justicia en el protocolo Código Infarto.

cual implica detenerse a pensar, explorar y discutir las diferentes etapas del proceso traslacional, que incluyen el descubrimiento científico, la implementación del binomio medicina basada en evidencias-medicina basada en valores igual que el anterior a través de guías de práctica clínica y su impacto en la población, principios clave para realizar una medicina de excelencia.

La ética reconoce a la justicia como uno de los principios básicos entre los individuos ${ }^{32}$ y cuando se incorpora en la bioética institucionalizada se convierte en una herramienta para implementar programas sanitarios. Un ejemplo son los programas de salud globales de la Organización Panamericana de la Salud, en los que la bioética se entiende esencialmente como un catalizador para la discusión y análisis de los problemas y los dilemas. ${ }^{33}$

En México, el presente artículo es el primero que analiza una estrategia de salud de la cardiología mexicana desde la bioética traslacional. El discernimiento de los valores, la gestión en salud y el conocimiento científico son claves para la eficiencia de este tipo de iniciativas y para lograr que la medicina transmita el valor de la vida.

\section{Agradecimientos}

Al ingeniero Rodrigo Campos Silva y Mercedes Ramos Velasco, por los elementos gráficos. Al
Instituto Mexicano del Seguro Social y al grupo transfuncional de ética, por las fructíferas discusiones.

\section{Conflicto de intereses}

Los autores declaran no tener conflicto de intereses alguno.

\section{Financiamiento}

Los autores no recibieron patrocinio para llevar a cabo este artículo.

\section{Responsabilidades éticas}

Protección de personas y animales. Los autores declaran que para esta investigación no se han realizado experimentos en seres humanos ni en animales.

Confidencialidad de los datos. Los autores declaran que en este artículo no aparecen datos de pacientes.

Derecho a la privacidad y consentimiento informado. Los autores declaran que en este artículo no aparecen datos de pacientes.

\section{Bibliografía}

1. Organisation for Economic Cooperation and Development [Internet]. Francia: Health at a Glance; 2019. 
2. Instituto Nacional de Estadística y Geografía. Características de las defunciones registradas en México durante 2017. México: Instituto Nacional de Estadística y Geografía; 2018.

3. Consejo Nacional de Evaluación de la Política de Desarrollo Social [Internet]. México: Evaluación estratégica de protección social; 2018.

4. Palomino P, Grande M, Linares M. The social determinants of health inequalities and exclusion in XX|st century societies. Rev Int Sociol. 2014;72:71-91

5. Gómez-Dantés O, Sesma S, Becerril V, Knaul F, Arreola H, Frenk J. Sistema de salud de México. Salud Publica Mex. 2011;53:s220-s232.

6. Shiguetomi-Medina J. La medicina traslacional: una disciplina emergente. Rev Mex Ortop Ped. 2013:15:64-67.

7. Borrayo G, Pérez G, Arriaga D, Martínez OG, Almeida, Ramírez E, et al Protocolo para atención de infarto agudo de miocardio en urgencias: Código Infarto. Rev Med Inst Mex Seg Soc. 2017:55:233-246.

8. Haynes RB, Sackett DL, Guyatt GH, Tugwell P. Clinical epidemiology, how to do clinical practice research. EE. UU.: Lippincott Williams \& Wilkins 2006.

9. Instituto Mexicano del Seguro Social [Internet]. México: El Código Infarto del IMSS, con la mayor cobertura en América Latina; 2016.

10. Arriaga J, Pérez G, Borrayo G. Dimensiones de calidad enfocadas en el protocolo de atención Código Infarto. Rev Med Inst Mex Seguro Soc. 2017:55:382-387.

11. Secretaría de Salud [Internet]. México: Sistemas de Información en Salud; 2020

12. Borrayo G, Madrid A, Arriaga R, Ramos MA, García J, Almeida E. Riesgo estratificado de los síndromes coronarios agudos. Resultados del primer Renasca-IMSS. Rev Med Inst Mex Seguro Soc 2010;48:259-264

13. Borrayo G, Rosas M, Ramírez E, Saturno G, Estrada J, Parra R, et al STEMI and NSTEMI: real-world Study in Mexico (RENASCA). Arch Med Res. 2018;49:609-619.

14. Ortiz-Flores J. El equilibrio reflexivo en el pensamiento bioético de Rodolfo Vázquez. México: México: Instituto de Ciencias Jurídicas/Universidad Nacional Autónoma de México; 2017

15. Trochim W, Kane C, Graham MJ, Pincus HA. Evaluating translational research: a process marker model. Clin Transl Sci. 2011;4:153-62

16. Peile E. Evidence-based medicine and values-based medicine: partners in clinical education as well as in clinical practice. BMC Med. 2013:11:40.
17. Sueiras P, Romano-Betech V, Vergil A, De Hoyos A, Quintana S, Ruddick W, et al. Today's medical self and the other: challenges and evolving solutions for enhanced humanization and quality of care. PLoS One. 2017; 12:e0181514.

18. Gracia D. Fundamentos de la bioética. España: Eudema; 1989.

19. Burrell G, Morgan G. Sociological paradigms and organisational analysis. Reino Unido: Ashgale Publishing; 1979.

20. Altamirano M, Garduño J, García MC Muñoz O, editores. Discernimiento de dilemas éticos en la práctica clínica. En: Ética clínica una perspectiva transfuncional. México: Corinter; 2006.

21. Cruz M. Medicina traslacional. Gac Med Mex. 2017;153:547-549.

22. Guerra LR. Por una bioética sin adjetivos II. Bioética, interdisciplinariedad y sociedad plural. ARS Med. 2008;37:137-146.

23. Olivé L. La bioética: Latinoamérica y la diversidad cultural. México: Instituto de Investigaciones Jurídicas/Universidad Nacional Autónoma de México; 2019.

24. Hostiuc S, Moldoveanu A, Dascalu ML. Unnthorsson R, Johanneson OI, Marcus I. Translational research the need of a new bioethics approach. J Transo Med. 2016,14:16.

25. Ley del Seguro Social. México: Diario Oficial de la Federación; 2019.

26. Contrato colectivo de trabajo 2019-2021. México: Sindicato Nacional de Trabajadores del Seguro Social/Instituto Mexicano del Seguro Social; 2019

27. Barnes J. The complete works of Aristotle. EE. UU.: Princeton/Bollingen Foundation; 1984

28. Consejo Nacional de Evaluación de la Política de Desarrollo Social [Internet]. México: Medición de la pobreza 2008-2018; 2019.

29. Haute Autorité de santé. Indicateurs de pratique clinique. Infarctus du myocarde (IDM); Francia: HAS; 2012. Disponible en: https://www. has-sante.fr/upload/docs/application/pdf/2012-07/04_indicateurs_idm_ actualisation_2012_vf.pdf

30. University of Ottawa Heart Institute [Internet]. STEMI Programa; 2014. Disponible en: https://www.ottawaheart.ca/healthcare-professionals/regional-national-programs/stemi-program

31. American Heart Association [Internet]. Mission: Lifeline; 2020.

32. López M. Ética profesional y complejidad: Ios principios y la religación. Perfiles Educativos. 2013;35:43-52.

33. Lolas F. La bioética en el contexto de los programas globales de salud. Pan Am J Public Health. 1999;6:65-68. 